\title{
The Sommerfeld Half-plane Problem Revisited, V: The Bifurcated Guide with Mixed Boundary Conditions on the Septum*
}

\author{
Albert E. Heins \\ Department of Mathematics, University of Michigan, Ann Arbor, Michigan 48109, U.S.A.
}

\begin{abstract}
We discuss the solution of the boundary value problem in a duct with a centered septum [9]. On the lower wall of the duct a Neumann condition is applied while on the upper wall a Dirichlet condition is applied. On the septum we apply a Dirichlet condition on the lower side and a Neumann condition on the upper one. This problem is formulated as a pair of integral equations of the Wiener-Hopf type for which we supply solutions for two modes of excitation as well as real and complex wave number. A critical examination is made of the construction, which reduces the problem to one in complex analysis. For real wave number, the physical parameters are provided in very simple forms.
\end{abstract}

\section{Introduction}

We continue our studies of parallel plate media with a discussion of a bifurcated duct problem recently considered by Lüneburg and Hurd [9]. They have studied problems for which the Neumann boundary condition was applied on the lower wall of a duct and the Dirichlet one on the upper wall. On the septum which is centred, the Dirichlet condition is applied on one side and the Neumann on the other. We note that there are two cases, and we shall only discuss one of them. In any event these problems lead to a coupled pair of Wiener-Hopf integral equations due to the conditions on the septum.

Such boundary value problems can be formulated effectively as integral equations of the Wiener-Hopf type with the aid of a Green's function whose boundary conditions are appropriate for the duct. But as we have long known, a Green's function for a duct with the boundary conditions we have described for the walls has various forms for the 'propagating terms'. Conventionally, we write a Green's function for the Helmholtz wave equation with outward-going propagating terms and attenuated ones from the source. If the wave number $k$ in the Helmholtz equation is real, the corresponding Green's function cannot be used to form a representation for the solution of the wave equation in the duct with a septum because we cannot evaluate

\footnotetext{
*From a paper presented at the conference on 'Wiener-Hopf Problems and Applications' held in Oberwolfach, West Germany, 20-26, July 1986.
} 
some of the integrals which arise from the Green's theorem. We have at this point two choices. One is to assume that the wave number $k$ has a small positive imaginary part and the 'propagating' and 'attenuated' wave numbers in the duct have negative real parts. In this case it is possible to give a representation but we are still left with the question about its interpretation when the imaginary part of $k$ vanishes.

A modified Green's function can be constructed by noting that a travelling wave term when $k$ is real, contains a solution of the homogeneous wave equation. We may add or subtract such solutions to the conventional Green's function and thereby only change its behaviour at infinity. The need for such a modified Green's function was noted by J. S. Schwinger in his study of duct problems in 1943 [11]. There he found it necessary to modify the travelling wave component of the Green's function when $k$ is real in a rather circuitous manner, in order to formulate the boundary value problems which he was examining. The only published account of this early work appeared in 1968 and it is woefully incomplete.

There is a second reason why we require either a positive imaginary part for $k$ in the conventional Green's function or a modified Green's function with appropriate conditions at infinity when $k$ is real. We are ultimately required to calculate the bilateral Fourier transform of the Green's function in order to apply the method of Wiener and Hopf. This transform should be analytic in the transform variable in some strip parallel to the real axis if we are to apply the method of Wiener and Hopf. When we use a complex $k$ with $\operatorname{Im} k>0$ this is possible. We will show in Section 2 how it is also possible to modify the conventional Green's function when $k$ is real and obtain a strip of analyticity. This strip, however, is not the same one we obtain when $k$ is complex.

A third issue arises when we attempt to form the Fourier transform of the integral equations which represent the boundary value problem we described. The convolution theorem is not directly applicable to the terms in these equations which contain normal derivatives of the Green's function which are evaluated on the septum. One way out of this difficulty is to find the Fourier transform of the representation which we give for $k$ real in Section 2 and then apply the boundary conditions. We regret that we did not make this point clear in [4] and [5]. A similar method should have been applied by Lüneburg and Hurd in [7]-[9].

We shall discuss the differences between the results in [9] and the present paper, noting among other matters, that we have found compact expressions for the magnitudes of the transmission coefficients. We also correct one of the graphs in [9]. Some of our calculations are similar to those used in [4] and we shall make free use of them.

\section{The formulation of two bifurcated duct problems}

Here we shall formulate two boundary value problems for the bifurcated duct which have been studied by Lüneburg and Hurd [9]. These boundary value problems will lead to coupled pairs of Wiener-Hopf integral equations and the solutions will be provided for $k$ real. To this end we require a representation for the solution $\phi(x, y)$ of the two-dimensional Helmholtz equation

$$
\phi_{x x}+\phi_{y y}+k^{2} \phi=0
$$

in the bifurcated duct with a centred septum which satisfies the following boundary 


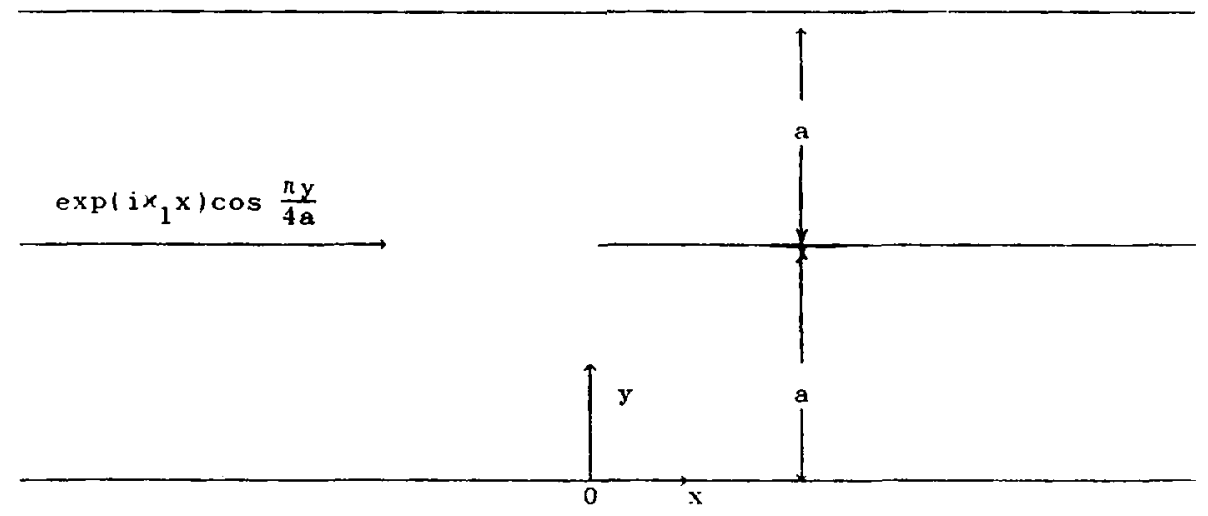

Fig. 1

conditions (see Fig. 1):

(i) $\phi_{y}=0,-\infty<x<\infty, y=0$

(ii) $\phi=0,-\infty<x<\infty, y=2 a$

(iii) $\phi=0,0<x<\infty, y=a-0$

(iv) $\phi_{y}=0,0<x<\infty, y=a+0$.

To these boundary conditions we add those conditions at infinity which pertain to the nature of the propagating modes which a duct can sustain. The first case we consider is the following. For $x \rightarrow-\infty, 0 \leqslant y \leqslant 2 a$

$$
\phi(x, y) \rightarrow \cos \frac{\pi y}{4 a}\left[\exp \left(\mathrm{i} \kappa_{1} x\right)+R_{1} \exp \left[-\mathrm{i} \kappa_{1} x\right],\right.
$$

where $\kappa_{1}=\sqrt{k^{2}-\pi^{2} / 16 a^{2}}>0$ and $\pi / 4<a k<3 \pi / 4$. That is, the larger duct can only sustain the lowest incident and reflected modes. For $x \rightarrow \infty, 0 \leqslant y \leqslant a$

$$
\phi(x, y) \rightarrow T_{1} \cos \frac{\pi y}{2 a} \exp \left(\mathrm{i} \kappa_{2} x\right),
$$

whereas for $x \rightarrow \infty, a \leqslant y \leqslant 2 a$

$$
\phi(x, y) \rightarrow T_{2} \sin \frac{\pi y}{2 a} \exp \left(\mathrm{i} \kappa_{2} x\right)
$$

that is, the lowest mode is transmitted in the small ducts. Here $\kappa_{2}=\sqrt{k^{2}-\pi^{2} / 4 a^{2}}>0$ and $\pi / 2<a k<3 \pi / 2$. Hence we have $\pi / 2<a k<3 \pi / 4$ in order that a single mode propagates in each duct.

In order to find the representation we seek, we use a Green's function for the duct $-\infty<x<\infty, 0 \leqslant y \leqslant 2 a$ which obeys the same boundary conditions as $\phi(x, y)$ on the walls of the duct and which obeys the same differential equation given in [2] and 
[3]. Such a Green's function $G\left(x, y ; x^{\prime}, y^{\prime}\right)$ is given by the infinite series

$$
\begin{aligned}
G\left(x, y ; x^{\prime}, y^{\prime}\right)= & \mathrm{i} \cos \frac{\pi y}{4 a} \cos \frac{\pi y^{\prime}}{4 a} \exp \left[\mathrm{i} \kappa_{1}\left|x-x^{\prime}\right| / 2 a \kappa_{1}\right] \\
& +\sum_{n=2}^{\infty} \cos \frac{(2 n-1) \pi y}{4 a} \cos \frac{(2 n-1) \pi y^{\prime}}{4 a} \\
& \times \exp \left[-\kappa_{2 n-1}\left|x-x^{\prime}\right| / 2 a \kappa_{2 n-1}\right.
\end{aligned}
$$

when $\pi / 4<a k<3 \pi / 4$ and $\kappa_{2 n-1}=\sqrt{\left[(2 n-1)^{2} \pi^{2} / 16 a^{2}\right]-k^{2}}>0, n=2,3, \ldots,$. Observe that this form of $G\left(x, y ; x^{\prime}, y^{\prime}\right)$ is bounded when $x^{\prime}$ or $x \rightarrow \pm \infty$. It is possible to modify the bounded term by adding a solution of the homogeneous Helmholtz equation which satisfies the boundary conditions on the wall of the duct and only change the behaviour of this Green's function at infinity. We add the term

$$
\frac{\left[-\mathrm{i} \cos \kappa_{1}\left(x-x^{\prime}\right)+\sin \kappa_{1}\left(x^{\prime}-x\right)\right]}{2 a \kappa_{1}} \cos \frac{\pi y}{4 a} \cos \frac{\pi y^{\prime}}{4 a},
$$

which is a solution of the homogeneous wave equation, to obtain

$$
\begin{aligned}
G^{(1)}\left(x, y ; x^{\prime}, y^{\prime}\right)= & {\left[\sin \kappa_{1}\left(x^{\prime}-x\right)-\sin \kappa_{1}\left|x^{\prime}-x\right|\right] \cos \frac{\pi y}{4 a} \cos \frac{\pi y^{\prime}}{4 a} / 2 a \kappa_{1} } \\
& +\sum_{n=2}^{\infty} \cos \frac{(2 n-1) \pi y}{4 a} \cos \frac{(2 n-1) \pi y^{\prime}}{4 a} \exp \left[-\kappa_{2 n-1}\left|x-x^{\prime}\right| / 2 a \kappa_{2 n-1}\right] .
\end{aligned}
$$

$G^{(1)}\left(x, y ; x^{\prime}, y^{\prime}\right)$ now has the property that it is bounded when $x^{\prime} \rightarrow-\infty$ and $O\left[\exp \left(-\kappa_{3} x^{\prime}\right)\right]$ when $x^{\prime} \rightarrow \infty$. In this form it is ideally suited to formulate the boundary value problem which we have described. The representation we now obtain by applying Green's integral theorem is

$$
\begin{aligned}
\phi(x, y)= & {\left[\exp \left(\mathrm{i} \kappa_{1} x\right)+R_{1} \exp \left(-\mathrm{i} \kappa_{1} x\right)\right] \cos \frac{\pi y}{4 a} } \\
& +\int_{0}^{\infty}\left[\left.G^{(1)}\left(x, y ; x^{\prime}, y^{\prime}\right) \frac{\partial \phi}{\partial y^{\prime}}\right|_{y^{\prime}=a-0}\right. \\
& \left.+\left.\phi(x, a+0) \frac{\partial G^{(1)}\left(x, y ; x^{\prime}, y^{\prime}\right)}{\partial y^{\prime}}\right|_{y^{\prime}=a}\right] \mathrm{d} x^{\prime} .
\end{aligned}
$$

Observe that we require $G^{(1)}\left(x, y ; x^{\prime}, y^{\prime}\right)$ in order to apply Green's theorem to Figure 1 with the behaviour of $\phi(x, y)$ at infinity which we have assumed. We cannot use $G\left(x, y ; x^{\prime}, y^{\prime}\right)$ when $k$ is real to obtain a representation since

$$
\lim _{x^{\prime} \rightarrow \infty}\left[\int_{0}^{a}+\int_{a}^{2 a}\right]\left[G\left(x, y ; x^{\prime}, y^{\prime}\right) \frac{\partial \phi\left(x^{\prime}, y^{\prime}\right)}{\partial x^{\prime}}-\phi\left(x^{\prime}, y^{\prime}\right) \frac{\partial G\left(x, y ; x^{\prime}, y^{\prime}\right)}{\partial y^{\prime}}\right] \mathrm{d} y^{\prime}
$$

does not exist. However, if $k$ is complex, has a positive imaginary part, and the $\kappa_{2 n-1}$, $n=2,3, \ldots$ have positive real parts, it is possible to formulate the problem with the aid of $G\left(x, y ; x^{\prime}, y^{\prime}\right)$. This however modifies the term exterior to the integral and the interpretation of the formulation is to be questioned when $k$ is real.

We have assumed that the integrals in (2.1) exist and therefore that the representation is valid. We will show that it is possible to produce the functions $\partial \phi /\left.\partial y\right|_{y=a-0}$, 
and $\left.\phi\right|_{y=a+0}, x>0$ which are bounded when $x \rightarrow \infty$ and are otherwise integrable when we invoke the boundary conditions on the system and solve the resulting coupled pair of Wiener-Hopf integral equations.

For a second example, we assume that for $x \rightarrow-\infty, 0 \leqslant y \leqslant 2 a$

$$
\phi(x, y) \rightarrow \cos \frac{\pi y}{4 a}\left[\exp \left(i \kappa_{1} x\right)+R_{1}^{\prime} \exp \left(-\mathrm{i} \kappa_{1} x\right)\right]+R_{3} \cos \frac{3 \pi y}{4 a} \exp \left(-\mathrm{i} \kappa_{3}^{\prime} x\right),
$$

where now $\kappa_{1}=\sqrt{k^{2}-\pi^{2} / 16 a^{2}}>0$ and $\kappa_{3}^{\prime}=\sqrt{k^{2}-9 \pi^{2} / 16 a^{2}}>0$ and $3 \pi / 4<a k<5 \pi / 4$. That is, the next reflected mode in the larger duct has been excited. For $x \rightarrow \infty, 0 \leqslant y \leqslant a$

$$
\phi(x, y) \rightarrow T_{1}^{\prime} \cos \frac{\pi y}{2 a} \exp \left(\mathrm{i} \kappa_{2} x\right),
$$

whereas for $x \rightarrow \infty, a \leqslant y \leqslant 2 a$

$$
\phi(x, y) \rightarrow T_{2}^{\prime} \sin \frac{\pi y}{2 a} \exp \left(\mathrm{i} \kappa_{2} x\right),
$$

with $\pi / 2<a k<3 \pi / 2$. Hence we have the inequality $3 \pi / 4<a k<5 \pi / 4$ for which there are two propagating modes in the large duct and one propagating mode in each of the smaller ducts. The formulation of this problem now entails a modification of $G^{(1)}\left(x, y ; x^{\prime}, y^{\prime}\right)$ to $G^{(2)}\left(x, y ; x^{\prime}, y^{\prime}\right)$. Here we have that

$$
\begin{aligned}
G^{(2)}\left(x, y ; x^{\prime}, y^{\prime}\right)= & {\left[\sin \kappa_{1}\left(x^{\prime}-x\right)-\sin \kappa_{1}\left|x^{\prime}-x\right|\right] \cos \frac{\pi y}{4 a} \cos \frac{\pi y^{\prime}}{4 a} / 2 a \kappa_{1} } \\
& +\left[\sin \kappa_{3}^{\prime}\left(x^{\prime}-x\right)-\sin \kappa_{3}\left|x^{\prime}-x\right|\right] \cos \frac{3 \pi y}{4 a} \cos \frac{3 \pi y^{\prime}}{4 a} / 2 a \kappa_{3}^{\prime} \\
& +\sum_{n=3}^{\infty} \frac{\cos (2 n-1) \pi y}{4 a} \frac{\cos (2 n-1) \pi y^{\prime}}{4 a} \\
& \times \exp \left[-\kappa_{2 n-1}\left|x^{\prime}-x\right| / 2 a \kappa_{2 n-1}\right],
\end{aligned}
$$

where now $\kappa_{2 n-1}>0, n=3,4, \ldots, \kappa_{3}^{\prime}=\sqrt{k^{2}-9 \pi^{2} / 16 a^{2}}>0$ and $G^{(2)}\left(x, y ; x^{\prime}, y^{\prime}\right)=$ $O\left[\exp \left(-\kappa_{5} x^{\prime}\right)\right]$ when $x^{\prime} \rightarrow \infty$. Hence the representation for $3 \pi / 4<a k<5 \pi / 4$ is

$$
\begin{aligned}
\phi(x, y)= & \cos \frac{\pi y}{4 a}\left[\exp \left(\mathrm{i} \kappa_{1} x\right)+R_{1}^{\prime} \exp \left(-\mathrm{i} \kappa_{1} x\right)\right]+R_{3} \cos \frac{3 \pi y}{4 a} \exp \left(-\mathrm{i} \kappa_{3}^{\prime} x\right) \\
& +\int_{0}^{\infty}\left[\left.G^{(2)}\left(x, y ; x^{\prime}, y^{\prime}\right) \frac{\partial \phi\left(x^{\prime}, y^{\prime}\right)}{\partial y^{\prime}}\right|_{y^{\prime}=a-0}\right. \\
& \left.+\left.\phi\left(x^{\prime}, y^{\prime}\right) \frac{\partial G^{(2)}\left(x, y ; x^{\prime}, y^{\prime}\right)}{\partial y^{\prime}}\right|_{y^{\prime}=a+0}\right] \mathrm{d} x^{\prime} .
\end{aligned}
$$

When we invoke the boundary conditions on the septum, we produce another coupled pair of integral equations of the Wiener-Hopf type.

If $k$ has a positive imaginary root as in [9], a representation similar to (2.1) and (2.2) can be obtained, save for the facts that there will be a different non-homogeneous term and $G^{(1)}\left(x, y ; x^{\prime}, y^{\prime}\right)$ and $G^{(2)}\left(x, y ; x^{\prime}, y^{\prime}\right)$ will be replaced by $G\left(x, y ; x^{\prime}, y^{\prime}\right)$. 


\section{The solution of the first problem}

The boundary conditions on the septum applied to (2.1) provide us with a coupled pair of Wiener-Hopf integral equations. We cannot, however, apply the convolution theorem directly to this system. This is due to the fact that we would be required to calculate the Fourier transforms of the $y$ and $y y^{\prime}$ derivatives of $G^{(1)}\left(x, y ; x^{\prime}, y^{\prime}\right)$ at $y=y^{\prime}=a$, which do not exist. We circumvent this difficulty by considering

$$
\begin{aligned}
\psi(x, y)= & \phi^{*}(x, y)+\int_{0}^{\infty}\left[\left.G^{(1)}\left(x, y, x^{\prime}, a\right) \frac{\partial \phi}{\partial y^{\prime}}\right|_{y^{\prime}=a-0}\right. \\
& \left.+\left.\phi\left(x^{\prime}, a+0\right) \frac{\partial G^{(1)}\left(x, y ; x^{\prime}, y^{\prime}\right)}{\partial y^{\prime}}\right|_{y^{\prime}=a}\right] \mathrm{d} x^{\prime}
\end{aligned}
$$

and its $y$ derivative for $y \neq a$. We define

$$
\begin{array}{rlrl}
\phi^{*}(x, y) & \equiv \cos \frac{\pi y}{4 a}\left[\exp \left(\mathrm{i} \kappa_{1} x\right)+R_{1} \exp \left(-\mathrm{i} \kappa_{1} x\right],\right. & & x>0, \\
& \equiv 0, & & x<0, \\
\phi(x, a-0) & \equiv 0, \quad x>0, & \\
\left.\frac{\partial \phi(x, y)}{\partial y}\right|_{y=a+0} \equiv 0, \quad x>0 . &
\end{array}
$$

Equation (3.1) contains the original problem for $x>0$ and therefore $\psi(x, a-0)=$ $\partial \psi(x, y) /\left.\partial y\right|_{y=a+0}=0$ there. Now $\psi(x, y)$ and $\partial \psi(x, y) / \partial y$ are $O\left[\exp \left(\kappa_{3} x\right)\right], x \rightarrow-\infty$. As we have noted, we seek a $\phi(x, y)$ and a $\partial \phi(x, y) / \partial y$ which are integrable for $x \geqslant 0$ and $O\left[\exp \left(i \kappa_{2} x\right)\right], x \rightarrow \infty$. Wiener-Hopf theory should now provide us with the unilateral Fourier transforms of $\phi(x, a+0)$ and $\left.\phi_{y}(x, y)\right|_{y=a-0}$, which should be analytic in the lower half-plane $\operatorname{Im} w<0$ and satisfy the Riemann-Lebesgue lemma. It should also give us the unilateral Fourier transforms of $\psi(x, a)$ and $\partial \psi(x, y) /\left.\partial y\right|_{y=a}$, $x \leqslant 0$, which should be analytic in the upper half-plane $\operatorname{Im} w>-\kappa_{3}$ and satisfy the Riemann-Lebesgue lemma there also.

The bilateral Fourier transform of $G^{(1)}\left(x, y ; 0, y^{\prime}\right)$ is

$$
\begin{aligned}
\hat{g}^{(1)}\left(w ; y, y^{\prime}\right) & =\int_{-\infty}^{\infty} \exp (-\mathrm{i} w x) G^{(1)}\left(x, y ; 0, y^{\prime}\right) \mathrm{d} x=\frac{\cos \sigma y \sin \sigma\left(2 a-y^{\prime}\right)}{\sigma \cos 2 \sigma a}, y<y^{\prime}, \\
& =\frac{\cos \sigma y^{\prime} \sin \sigma(2 a-y)}{\sigma \cos 2 \sigma a}, y>y^{\prime} .
\end{aligned}
$$

Since $G^{(1)}\left(x, y ; 0, y^{\prime}\right)=O\left[\sin \kappa_{1} x\right], x \rightarrow \infty$ and $O\left[\exp \left(\kappa_{3} x\right)\right], x \rightarrow-\infty, \hat{g}^{(1)}\left(w ; y, y^{\prime}\right)$ is analytic in the strip $-\kappa_{3}<\operatorname{Im} w<0$ and obeys the Riemann-Lebesgue lemma there. For $y \neq y^{\prime}$, the $y$ and $y y^{\prime}$ derivatives also satisfy this lemma. Now, based upon the expected behaviour of $\phi(x, a+0)$ and $\partial \phi /\left.\partial y\right|_{y=a-0}$, these functions should have unilateral Fourier transforms, analytic in the lower half-plane $\operatorname{Im} w<0$, whereas $\psi(x, a)$ and $\partial \psi(x, y) /\left.\partial y\right|_{y=a}$ should have unilateral Fourier transforms analytic in the upper half-plane $\operatorname{Im} w>-\kappa_{3}$.

It follows that since the transforms of $\phi(x, a+0), \partial \phi(x, y) /\left.\partial y\right|_{y=a-0}, \psi(x, a)$, $\partial \psi(x, y) /\left.\partial y\right|_{y=a}, \phi^{*}(x, y)$ and $G^{(1)}\left(x, y ; 0, y^{\prime}\right)$ would have a common strip of analyticity, 
that is $-\kappa_{3}<\operatorname{Im} w<0$, we have for $y \neq a$

$$
\begin{aligned}
\hat{\psi}(w, y)= & {\left[\frac{1}{\mathrm{i}\left(w-\kappa_{1}\right)}+\frac{R_{1}}{\mathrm{i}\left(w+\kappa_{1}\right)}\right] \cos \frac{\pi y}{4 a} } \\
& +\left[\frac{\sin \sigma a}{\sigma \cos 2 \sigma a} \hat{\phi}_{y}(w)-\frac{\cos \sigma a}{\cos 2 \sigma a} \hat{\phi}(w)\right] \cos \sigma y, \quad 0<y<a
\end{aligned}
$$

and

$$
\begin{aligned}
\hat{\psi}(w, y)= & {\left[\frac{1}{\mathrm{i}\left(w-\kappa_{1}\right)}+\frac{R_{1}}{\mathrm{i}\left(w+\kappa_{1}\right)}\right] \cos \frac{\pi y}{4 a} } \\
& +\left[\frac{\cos \sigma a}{\sigma \cos 2 \sigma a} \hat{\phi}_{y}(w)-\frac{\sin \sigma a}{\cos 2 \sigma a} \hat{\phi}(w)\right] \sin \sigma(2 a-y), \quad a<y<2 a .
\end{aligned}
$$

Here $\sigma=\left(k^{2}-w^{2}\right)^{1 / 2}$ and is taken to be real and positive when $|\operatorname{Re} w|<k, \operatorname{Im} w=0$, and

and

$$
\begin{aligned}
\hat{\phi}(w) & =\int_{0}^{\infty} \exp (-\mathrm{i} w x) \phi(x, a+0) \mathrm{d} x, \\
\hat{\phi}_{y}(w) & =\left.\int_{0}^{\infty} \exp (-\mathrm{i} w x) \frac{\partial \phi(x, y)}{\partial y}\right|_{a-0} \mathrm{~d} x
\end{aligned}
$$

$$
\hat{\psi}(w, y)=\int_{-\infty}^{0} \exp (-\mathrm{i} w x) \psi(x, y) \mathrm{d} x .
$$

We note that as a consequence of the edge condition that $\phi(x, a+0)$ should be $O\left(x^{1 / 4}\right), x \rightarrow 0+$ and $\partial \phi(x, a-0) / \partial y$ should be $O\left(x^{-3 / 4}\right), x \rightarrow 0+$ and therefore $\hat{\phi}(w)=O\left(w^{-5 / 4}\right)$ and $\hat{\phi}_{y}(w)=O\left(w^{-1 / 4}\right),|w| \rightarrow \infty$, Im $w<0$. Hence $\hat{\psi}(w, a)$ would then be $O\left(w^{-1}\right), \quad \operatorname{Im} w>-\kappa_{3}, \quad|w| \rightarrow \infty$ and therefore would obey the Riemann-Lebesgue lemma, and so would $\partial \hat{\psi}(w, y) / \partial y$. However, we do not require these facts since they are derivable from our analysis.

Now it is possible to take the limit $y \rightarrow a-0$ in (3.2) and $y \rightarrow a+0$ for the $y$ derivative of (3.3). We have in matrix notation

where

$$
\begin{aligned}
& \mathbf{A}(w) \boldsymbol{\Phi}(w)-\frac{1}{\mathrm{i} \sqrt{2}}\left[\frac{1}{w-\kappa_{1}}+\frac{R_{1}}{w+\kappa_{1}}\right]\left(\begin{array}{c}
\frac{\pi}{4 a} \\
-1
\end{array}\right)=\boldsymbol{\Psi}(w) \\
& \mathbf{A}(w)=\left(\begin{array}{cc}
\frac{\cos ^{2} \sigma a}{\cos 2 \sigma a} & -\frac{\sigma \sin \sigma a \cos \sigma a}{\cos 2 \sigma a} \\
-\frac{\sin \sigma a \cos \sigma a}{\sigma \cos 2 \sigma a} & \frac{\cos ^{2} \sigma a}{\cos 2 \sigma a}
\end{array}\right) \\
& \mathbf{\Phi}(w)=\left(\begin{array}{c}
\hat{\phi}_{y}(w) \\
\hat{\phi}(w)
\end{array}\right) \text { and } \Psi(w)=\left(\begin{array}{c}
\hat{\psi}_{y}(w, a) \\
\hat{\psi}(w, a)
\end{array}\right)
\end{aligned}
$$

From this point onward, we have a function-theoretic problem whose general 
features we have described in [2] and [3]-[5]. We write

$$
\mathbf{A}(w)=\frac{\cos \sigma a}{\sqrt{\cos 2 \sigma a}}\left(\begin{array}{cc}
\frac{\cos \sigma a}{\sqrt{\cos 2 \sigma a}} & -\frac{\sigma \sin \sigma a}{\sqrt{\cos 2 \sigma a}} \\
-\frac{\sin \sigma a}{\sigma \sqrt{\cos 2 \sigma a}} & \frac{\cos \sigma a}{\sqrt{\cos 2 \sigma a}}
\end{array}\right) .
$$

The scalar factor can be rewritten as

$$
\frac{\cos \sigma a}{\sqrt{\cos 2 \sigma a}}=\frac{K_{-}(w)}{K_{+}(w)},
$$

where $K_{-}(w)$ and its reciprocal are analytic in the lower half-plane $\operatorname{Im} w<0$ and have algebraic behaviour at infinity in this half-plane, whereas $K_{+}(w)$ has the same properties in the upper half-plane $\operatorname{Im} w>-\kappa_{3}$.

We have discussed the factorization of scalar terms of this type on several occasions ([2], [4], [5]). Here we give the final result for the case at hand.

$$
\begin{aligned}
K_{-}(w)= & \frac{a}{\pi} \frac{\left(w^{2}-\kappa_{2}^{2}\right)}{\sqrt{w^{2}-\kappa_{1}^{2}}} \exp \left(-\frac{\mathrm{i} a w \ln 2}{\pi}\right) \\
& \times \prod_{n=2}^{\infty} \frac{\left[\sqrt{1-\frac{4 a^{2} k^{2}}{\pi^{2}(2 n-1)^{2}}}+\frac{2 \mathrm{i} a w}{\pi(2 n-1)}\right]}{\sqrt{\sqrt{1-\frac{16 a^{2} k^{2}}{\pi^{2}(2 n-1)^{2}}}}+\frac{4 \mathrm{i} a w}{\pi(2 n-1)}}
\end{aligned}
$$

and

$$
\frac{1}{K_{+}(w)}=\exp \left(\frac{\mathrm{i} a w \ln 2}{\pi}\right) \prod_{n=2}^{\infty} \frac{\left[\sqrt{1-\frac{4 a^{2} k^{2}}{\pi^{2}(2 n-1)^{2}}}-\frac{2 \mathrm{i} a w}{\pi(2 n-1)}\right]}{\sqrt{\sqrt{1-\frac{16 a^{2} k^{2}}{\pi^{2}(2 n-1)^{2}}}-\frac{4 \mathrm{i} a w}{\pi(2 n-1)}}}
$$

The factors $\exp ( \pm(\mathrm{i} a w \ln 2 / \pi))$ have been inserted to render the terms $K_{-}(w)$ and $K_{+}(w)$ of algebraic growth. Indeed $K_{-}(w)$ and $K_{+}(w)$ are $O\left(w^{1 / 2}\right),|w| \rightarrow \infty$ in their respective half-planes of analyticity [10]. The corresponding terms in [9] have to be modified with terms $\exp ( \pm(\mathrm{i} a w \ln 2 / \pi))$ or it will not be possible to apply the Liouville theorem.

The matrix in (3.4b) can be rewritten as

$$
\begin{aligned}
\mathbf{B}(w) & =\left(\begin{array}{cr}
\frac{\cos \sigma a}{\sqrt{\cos 2 \sigma a}} & -\frac{\sigma \sin \sigma a}{\sqrt{\cos 2 \sigma a}} \\
-\frac{\sin \sigma a}{\sigma \sqrt{\cos 2 \sigma a}} & \frac{\cos \sigma a}{\sqrt{\cos 2 \sigma a}}
\end{array}\right) \\
& =\exp \left(\begin{array}{cc}
0 & \sigma \\
1 / \sigma & 0
\end{array}\right) \ln \sqrt{\frac{\cos \left(\sigma a+\frac{\pi}{4}\right)}{\cos \left(\sigma a-\frac{\pi}{4}\right)}}
\end{aligned}
$$


We require the additive decomposition of the matrix in the exponent such that one term is analytic in the lower half-plane $\operatorname{Im} w<0$, whereas the other is analytic in the upper half-plane $\operatorname{Im} w>-\kappa_{3}$. We note that $\cos (\sigma \mathrm{a}+(\pi / 4))$ has two real zeros at $w= \pm \kappa_{1}$, whereas $\cos (\sigma a-(\pi / 4))$ has two imaginary zeros at $w= \pm \mathrm{i} \kappa_{3}$. We accomplish this decomposition as we did in [4] and [5] with the aid of Cauchy's integral theorem with a path of integration drawn parallel to the real axis in the strip of analyticity. This then gives

$$
\exp \left(\begin{array}{cc}
0 & \sigma \\
1 / \sigma & 0
\end{array}\right) \ln h_{+}(w) \exp \left(\begin{array}{cc}
0 & \sigma \\
1 / \sigma & 0
\end{array}\right) \ln h_{-}(w)
$$

which is possible since the matrices obviously commute. $\left[\ln h_{-}(w)\right] / \sigma(w)$ is analytic in the lower half-plane $\operatorname{Im} w<0$, whereas $\left[\ln h_{+}(w)\right] / \sigma(w)$ is analytic in the upper half-plane $\operatorname{Im} w>-\kappa_{3}$. Thus the component of (3.5) which is analytic in the upper half-plane $\operatorname{Im} w>-\kappa_{3}$ is

and

$$
\mathbf{B}_{+}(w)=\left(\begin{array}{cc}
\frac{1}{2}\left[h_{+}(w)+\frac{1}{h_{+}(w)}\right] & \frac{\sigma(w)}{2}\left[h_{+}(w)-\frac{1}{h_{+}(w)}\right] \\
\frac{1}{2 \sigma(w)}\left[h_{+}(w)-\frac{1}{h_{+}(w)}\right] & \frac{1}{2}\left[h_{+}(w)+\frac{1}{h_{+}(w)}\right]
\end{array}\right),
$$

$$
\mathbf{B}_{-}(w)=\left(\begin{array}{cc}
\frac{1}{2}\left[h_{-}(w)+\frac{1}{h_{-}(w)}\right] & \frac{\sigma(w)}{2}\left[h_{-}(w)-\frac{1}{h_{-}(w)}\right] \\
\frac{1}{2 \sigma(w)}\left[h_{-}(w)-\frac{1}{h_{-}(w)}\right] & \frac{1}{2}\left[h_{-}(w)+\frac{1}{h_{-}(w)}\right]
\end{array}\right)
$$

has entries which are analytic in the lower half-plane $\operatorname{Im} w<0$. We shall give the explicit form of $h_{+}(w)$ since this is the only term we use. We have

$$
\begin{aligned}
& h_{+}(w)=\left[\frac{w \mathrm{i}-\sigma(w)}{w \mathrm{i}+\sigma(w)} \frac{k^{2}+w \mathrm{i} \kappa_{3}+\sigma(w) \sigma\left(\mathrm{i} \kappa_{3}\right)}{k^{2}+w i \kappa_{3}-\sigma(w) \sigma\left(\mathrm{i} \kappa_{3}\right)}\right. \\
& \times \prod_{n=2}^{\infty} \frac{k^{2}+w \mathrm{i} \kappa_{4 n-3}-\sigma(w) \sigma\left(\mathrm{i} \kappa_{4 n-3}\right)}{k^{2}+w i \kappa_{4 n-3}+\sigma(w) \sigma\left(\mathrm{i} \kappa_{4 n-3}\right)} \\
&\left.\times \frac{k^{2}+w i \kappa_{4 n-1}+\sigma(w) \sigma\left(\mathrm{i} \kappa_{4 n-1}\right)}{k^{2}+w i \kappa_{4 n-1}-\sigma(w) \sigma\left(\mathrm{i} \kappa_{4 n-1}\right)}\right]^{1 / 4}
\end{aligned}
$$

In a fashion which we have made familiar in [1]-[5], we can derive two relations by a Wiener-Hopf separation from (3.4), that is

$$
\begin{aligned}
K_{-}(w) \mathbf{B}_{-}(w) \boldsymbol{\Phi}(w) & -\frac{1}{\mathrm{i} \sqrt{2}}\left[\frac{K_{+}\left(\kappa_{1}\right) h_{+}\left(\kappa_{1}\right)}{w-\kappa_{1}}+\frac{R_{1} K_{+}\left(-\kappa_{1}\right) h_{+}\left(-\kappa_{1}\right)}{w+\kappa_{1}}\right] \\
& \times\left(\begin{array}{c}
\frac{\pi}{4 a} \\
-1
\end{array}\right)=\left(\begin{array}{c}
\varepsilon_{1}(w) \\
\varepsilon_{2}(w)
\end{array}\right)
\end{aligned}
$$


and

$$
\begin{aligned}
& K_{+}(w) \mathbf{B}_{+}^{-1}(w) \Psi(w)+\frac{1}{\mathrm{i} \sqrt{2}}\left[\frac{K_{+}(w) \mathbf{B}_{+}^{-1}(w)-K_{+}\left(\kappa_{1}\right) \mathbf{B}_{+}^{-1}\left(\kappa_{1}\right)}{w-\kappa_{1}}\right. \\
& \left.\quad+R_{1} \frac{\left\{K_{+}(w) \mathbf{B}_{+}^{-1}(w)-K_{+}\left(-\kappa_{1}\right) \mathbf{B}_{+}^{-1}\left(-\kappa_{1}\right)\right\}}{w+\kappa_{1}}\right]\left(\begin{array}{c}
\frac{\pi}{4 a} \\
-1
\end{array}\right)=\left(\begin{array}{l}
\varepsilon_{1}(w) \\
\varepsilon_{2}(w)
\end{array}\right),
\end{aligned}
$$

where $\varepsilon_{1}(w)$ and $\varepsilon_{2}(w)$ are entire functions. Now we know that $h_{+}(w)=O\left(w^{1 / 4}\right)$, $|w| \rightarrow \infty, \operatorname{Im} w>-\kappa_{3}$ and $h_{-}(w)=O\left(w^{-1 / 4}\right),|w| \rightarrow \infty, \operatorname{Im} w<0$. We know that the scalar factors are both $O\left(w^{1 / 2}\right),|w| \rightarrow \infty$ in their half-planes of regularity upon using familiar methods [10]. Hence, since we seek a $\Phi(w)$ and a $\Psi(w)$ which obey the Riemann--Lebesgue lemma in their respective half-planes of analyticity, we find that $\left|\varepsilon_{1}(w)\right|<M_{1}|w|^{7 / 4}+M_{2}|w|^{3 / 4}$ and $\left|\varepsilon_{2}(w)\right|<M_{3}|w|^{3 / 4}$ for fixed $M_{1}, M_{2}$ and $M_{3}$ when $|w| \rightarrow \infty$. This implies [6, p. 204] that $\varepsilon_{1}(w)=\alpha+\beta w$ and $\varepsilon_{2}(w)=\gamma$ where $\alpha, \beta$ and $\gamma$ are constants. When we solve for $\Phi(w)$ in (3.8), we find two terms which are of the order $w^{5 / 4}$ and $w^{1 / 4}$ when $|w| \rightarrow \infty, \operatorname{Im} w<0$. In order that $\Phi(w)$ obey the Riemann-Lebesgue lemma we are required to choose $\beta=\gamma=0$ and hence $\varepsilon_{1}(w)=\alpha$ and $\varepsilon_{2}(w) \equiv 0$. Notice that we have not employed the edge condition. Now $\hat{\phi}(w)=O\left(w^{-5 / 4}\right)$ and $\hat{\phi}_{y}=O\left(w^{-1 / 4}\right)$ when $|w| \rightarrow \infty, \operatorname{Im} w<0$ while $\hat{\psi}_{y}=O\left(w^{-1 / 4}\right)$ and $\hat{\psi}_{y}=O\left(w^{-1}\right)$ when $|w| \rightarrow \infty, \operatorname{Im} w>-\kappa_{3}$.

The arbitrary constant $\alpha$ is eliminated on physical grounds. We have formulated the duct problem under the assumption that there are no incident duct modes from the right upon the discontinuity due to the presence of the semi-infinite septum. Hence $K_{-}(w)$, which has a zero at $w=-\kappa_{2}$, would produce such a wave unless there is a zero on the right-hand side of (3.7). This gives us

$$
\frac{1}{\mathrm{i} \sqrt{2}}\left[-\frac{K_{+}\left(\kappa_{1}\right) h_{+}\left(\kappa_{1}\right)}{\kappa_{1}+\kappa_{2}}+\frac{R_{1} K_{+}\left(-\kappa_{1}\right) h_{+}\left(-\kappa_{1}\right)}{\kappa_{1}-\kappa_{2}}\right]\left(\begin{array}{c}
\frac{\pi}{4 a} \\
-1
\end{array}\right)+\left(\begin{array}{l}
\alpha \\
0
\end{array}\right)=0
$$

It follows that $\alpha=0$ and

$$
R_{1}=\frac{\kappa_{1}-\kappa_{2}}{\kappa_{1}+\kappa_{2}} \frac{K_{+}\left(\kappa_{1}\right) h_{+}\left(\kappa_{1}\right)}{K_{+}\left(-\kappa_{1}\right) h_{+}\left(-\kappa_{1}\right)} .
$$

But $K_{+}\left(\kappa_{1}\right) h_{+}\left(\kappa_{1}\right)$ is conjugate to $K_{+}\left(-\kappa_{1}\right) h_{+}\left(-\kappa_{1}\right)$ and therefore

$$
\left|R_{1}\right|=\frac{\kappa_{1}-\kappa_{2}}{\kappa_{1}+\kappa_{2}}, \frac{\pi}{2}<a k<\frac{3 \pi}{4} .
$$

In order to determine $T_{1}$ and $T_{2}$, the amplitudes of the transmitted waves in the small ducts, we write (3.1) as a Fourier integral and note that it is necessary to distinguish the cases $y<a$ and $y>a$. For $y<a$

$$
\begin{aligned}
\psi(x, y)= & \phi^{*}(x, y)+\frac{1}{2 \pi} \int_{\mathscr{L}} \frac{\exp (\mathrm{iw} x) \cos \sigma y}{\cos 2 \sigma a} \\
& \times\left[\frac{\sin \sigma a \hat{\phi}_{y}(w)}{\sigma}-\cos \sigma a \hat{\phi}(w)\right] \mathrm{d} w,
\end{aligned}
$$

where $\mathscr{L}$ is a path drawn parallel to the real axis in the strip $-\kappa_{\mathbf{3}}<\operatorname{Im} w<0$. Upon 
inserting the expressions we have derived for $\hat{\phi}_{y}(w)$ and $\hat{\phi}(w)$, we obtain

$$
\begin{aligned}
\psi(x, y)= & \phi^{*}(x, y)-\frac{1}{4 \pi \mathrm{i} \sqrt{2}} \int_{\mathscr{L}} \frac{\cos \sigma y \exp (\mathrm{i} w x) \mathrm{d} w}{\cos \sigma a K_{+}(w)} \\
& \times\left[\left(1-\frac{\pi}{4 a \sigma}\right) h_{+}(w)+\left(1+\frac{\pi}{4 a \sigma}\right) \frac{1}{h_{+}(w)}\right] \\
& \times\left[\frac{K_{+}\left(\kappa_{1}\right) h_{+}\left(\kappa_{1}\right)}{w-\kappa_{1}}+\frac{R_{1} K_{+}\left(-\kappa_{1}\right) h_{+}\left(-\kappa_{1}\right)}{w+\kappa_{1}}\right] .
\end{aligned}
$$

We have expressed the integrand in terms of $h_{+}(w)$ and $K_{+}(w)$ since we shall close the path $\mathscr{L}$ with a sequence of semicircles which pass between the zeros of $\cos \sigma a$ in the upper half-plane $\operatorname{Im} w>-\kappa_{3}$ when $x>0$. Under these circumstances, $h_{+}(w)$ and $K_{+}(w)$ can be evaluated directly and the only residues which arise come from $\kappa_{1},-\kappa_{1}$ and the zeros of $\cos \sigma a$. In view of the fact that $h_{+}(w)+1 / h_{+}(w)$ and $\left[h_{+}(w)-1 / h_{+}(w)\right] / \sigma(w)$ are single-valued analytic functions of $w$ in the upper half-plane $\operatorname{Im} w>-\kappa_{3}$, we may close the path $\mathscr{L}$ as we have just described it and obtain for $x \rightarrow \infty, 0<y<a$

$$
\psi(x, y) \rightarrow-\frac{4 \sqrt{2} \kappa_{1} K_{+}\left(\kappa_{1}\right) h_{+}\left(\kappa_{1}\right)}{3 \pi}\left[h_{+}\left(\kappa_{2}\right)+\frac{3}{h_{+}\left(\kappa_{2}\right)}\right] \cos \frac{\pi y}{2 a} \exp \left(i \kappa_{2} x\right) .
$$

Upon noting that $\left|h_{+}\left(\kappa_{1}\right)\right|=(\pi / 2)^{1 / 4},\left|h_{+}\left(\kappa_{2}\right)\right|=3^{1 / 4},\left|K_{+}\left(\kappa_{1}\right)\right|=3^{1 / 2} \pi^{1 / 4} / 2^{5 / 4}$ and $\left|K_{+}\left(\kappa_{2}\right)\right|=3^{1 / 4}(2 / \pi)^{1 / 2}$, the magnitude of $T_{1}$ which is the coefficient of $\cos \frac{\pi y}{2 a} \exp \left(i \kappa_{2} x\right)$ in $(3.8)$ reduces to

$$
\frac{\kappa_{1}[4+2 \sqrt{3} \cos 2 v]^{1 / 2}}{\left(\kappa_{1}+\kappa_{2}\right)}
$$

where $v=\arg h_{+}\left(\kappa_{2}\right)$. In a similar fashion

$$
\left|T_{2}\right|=\frac{\kappa_{1}[4-2 \sqrt{3} \cos 2 v]^{1 / 2}}{\kappa_{1}+\kappa_{2}} .
$$

We note that $\left|R_{1}\right|,\left|T_{1}\right|$ and $\left|T_{2}\right|$ satisfy the conservation condition

$$
\left(1-\left|R_{1}\right|^{2}\right) \kappa_{1}=\kappa_{2}\left[\left|T_{1}\right|^{2}+\left|T_{2}\right|^{2}\right] / 2 \text {. }
$$

This then gives us the reflection coefficient

$$
\left|R_{1}\right|=\frac{\kappa_{1}-\kappa_{2}}{\kappa_{1}+\kappa_{2}}
$$

and the two transmission coefficients

and

$$
\sqrt{\frac{\kappa_{2}}{\kappa_{1}}}\left|T_{1}\right|=\frac{\left\{\kappa_{1} \kappa_{2}[4+2 \sqrt{3} \cos 2 \nu]\right\}^{1 / 2}}{2^{1 / 2}\left(\kappa_{1}+\kappa_{2}\right)}
$$

$$
\sqrt{\frac{\kappa_{2}}{\kappa_{1}}}\left|T_{2}\right|=\frac{\left\{\kappa_{1} \kappa_{2}[4-2 \sqrt{3} \cos 2 v]\right\}^{1 / 2}}{2^{1 / 2}\left(\kappa_{1}+\kappa_{2}\right)} \text {. }
$$




\section{The solution of the second problem}

Upon following the ideas developed in Section 3, we now obtain for the second problem in Section 2, the counterpart to equation (3.4):

$$
\begin{aligned}
& -\mathbf{A}(w) \boldsymbol{\Phi}^{\prime}(w)-\frac{1}{\mathrm{i} \sqrt{2}}\left[\frac{1}{w-\kappa_{1}}+\frac{R_{1}^{\prime}}{\left(w+\kappa_{1}\right)}\right]\left(\begin{array}{c}
\frac{\pi}{4 a} \\
-1
\end{array}\right) \\
& -\frac{R_{3}}{\mathrm{i} \sqrt{2}\left(w+\kappa_{3}^{\prime}\right)}\left(\begin{array}{c}
\frac{3 \pi}{4 a} \\
1
\end{array}\right)=\Psi^{\prime}(w) .
\end{aligned}
$$

Now $G\left(x, y ; x^{\prime}, y^{\prime}\right)$ has been modified to $G^{(2)}\left(x, y ; x^{\prime}, y^{\prime}\right)$, and the bilateral Fourier transform of the latter is now valid in the strip $-\kappa_{5}<\operatorname{Im} w<0$ and therefore $\Psi^{\prime}(w)$ will be analytic in the upper half-plane $\operatorname{Im} w>-\kappa_{5}$. Although $\kappa_{3}^{\prime}$ is real we can still write $\mathbf{A}(w)=K^{\prime}(w) \mathbf{B}(w)$. The Wiener-Hopf factors of the scalar term $K(w)$ and the matrix $\mathbf{B}(w)$ are now modified. In this case we have that

$$
\begin{aligned}
K_{-}^{\prime}(w)= & \frac{3}{4} \frac{\left[\sqrt{1-\frac{4 a^{2} k^{2}}{\pi^{2}}}+\frac{2 \mathrm{i} a w}{3 \pi}\right]\left(w^{2}-\kappa_{2}^{2}\right) \exp \left[-\frac{\mathrm{i} a w}{\pi}\left(\ln 2+\frac{2}{3}\right)\right]}{\sqrt{\left[w^{2}-\kappa_{1}^{2}\right]\left(w^{2}-\kappa_{3}^{2}\right)}} \\
& \times \prod_{n=3}^{\infty} \frac{\left[\sqrt{1-\frac{4 a^{2} k^{2}}{(2 n-1)^{2} \pi^{2}}}+\frac{2 \mathrm{i} a w}{\pi(2 n-1)}\right]}{\sqrt{\sqrt{1-\frac{16 a^{2} k^{2}}{\pi^{2}(2 n-1)^{2}}}+\frac{4 i a w}{\pi(2 n-1)}}}
\end{aligned}
$$

and its reciprocal are analytic in the lower half-plane $\operatorname{Im} w<0$. Further

$$
\begin{aligned}
& \frac{1}{K_{+}^{\prime}(w)}=\left[\sqrt{1-\frac{4 a^{2} k^{2}}{9 \pi^{2}}}-\frac{2 \mathrm{i} a w}{3 \pi}\right] \exp \left[\frac{\mathrm{i} a w}{\pi}\left(\ln 2+\frac{2}{3}\right)\right] \\
& \times \prod_{n=3}^{\infty} \frac{\left[\sqrt{1-\frac{4 a^{2} k^{2}}{\pi^{2}(2 n-1)^{2}}}-\frac{2 \mathrm{i} a w}{\pi(2 n-1)}\right]}{\sqrt{\sqrt{1-\frac{16 a^{2} k^{2}}{\pi^{2}(2 n-1)^{2}}}-\frac{4 i a w}{\pi(2 n-1)}}}
\end{aligned}
$$

and its reciprocal are analytic in the upper half-plane $\operatorname{Im} w>-\kappa_{5} . K_{ \pm}^{\prime}(w)=O(1)$ when $|w| \rightarrow \infty$ in their respective half-planes of analyticity.

Now, as for the factorizing of $\mathbf{B}(w)$, we obtain in this case matrices of the form (3.6) and (3.7), but their elements $h_{ \pm}(w)$ are now replaced by $h_{ \pm}^{\prime}(w)$, where

$$
\begin{aligned}
h_{+}^{\prime}(w)= & {\left[\sum_{n=2}^{\infty} \frac{k^{2}+w i \kappa_{4 n-3}-\sigma(w) \sigma\left(i \kappa_{4 n-3}\right)}{k^{2}+w i \kappa_{4 n-3}+\sigma(w) \sigma\left(i \kappa_{4 n-3}\right)}\right.} \\
& \left.\times \frac{k^{2}+w i \kappa_{4 n-1}+\sigma(w) \sigma\left(i \kappa_{4 n-1}\right)}{k^{2}+w i \kappa_{4 n-1}-\sigma(w) \sigma\left(\mathrm{i} \kappa_{4 n-1}\right)}\right]^{1 / 4} .
\end{aligned}
$$


This has been derived by the method employed in [4] and [5], and it is analytic in the upper half-plane $\operatorname{Im} w>-\kappa_{5}$ where we have chosen the branch which is 1 when $w= \pm k$. We do not require $h_{-}^{\prime}(w)$ explicitly. We also have $h_{+}^{\prime}(w)=O\left(w^{1 / 4}\right),|w| \rightarrow \infty$, $\operatorname{Im} w<-\kappa_{5}$ and $h_{-}^{\prime}(w)=O\left(w^{-1 / 4}\right),|w| \rightarrow \infty, \operatorname{Im} w<0$.

We now apply the method of Wiener and Hopf to equation (4.1) to obtain

$$
\begin{aligned}
& K_{-}^{\prime}(w) \mathbf{B}_{-}^{\prime}(w) \Phi^{\prime}(w)+\frac{1}{i \sqrt{2}}\left[\frac{K_{+}^{\prime}\left(\kappa_{1}\right) h_{+}^{\prime}\left(\kappa_{1}\right)}{w-\kappa_{1}}+\frac{R_{1}^{\prime} K_{+}^{\prime}\left(-\kappa_{1}\right) h_{+}^{\prime}\left(-\kappa_{1}\right)}{w+\kappa_{1}}\right]\left(\begin{array}{c}
\frac{\pi}{4 a} \\
-1
\end{array}\right) \\
& \quad+\frac{1}{i \sqrt{2}}\left[\frac{K_{+}^{\prime}\left(-\kappa_{3}\right)}{h_{+}^{\prime}\left(-\kappa_{3}\right)} \frac{R_{3}}{\left(w+\kappa_{3}^{\prime}\right)}\right]\left(\begin{array}{c}
\frac{3 \pi}{4 a} \\
1
\end{array}\right)=\left(\begin{array}{l}
\varepsilon_{1}^{\prime}(w) \\
\varepsilon_{2}^{\prime}(w)
\end{array}\right),
\end{aligned}
$$

where $\varepsilon_{1}^{\prime}(w)$ and $\varepsilon_{2}^{\prime}(w)$ are entire functions.

We have seen in Section 3 that an application of the Liouville extended theorem $\left[6, p\right.$. 204] gives us $\varepsilon_{1}^{\prime}(w)=\alpha^{\prime}+\beta^{\prime} w$ and $\varepsilon_{2}^{\prime}(w)=\gamma^{\prime}$ where $\alpha^{\prime}, \beta^{\prime}$ and $\gamma^{\prime}$ are constants. If we solve for $\Phi^{\prime}(w)$ we find that there are terms $O\left(w^{5 / 4}\right), O\left(w^{3 / 4}\right)$ and $O\left(w^{1 / 4}\right),|w| \rightarrow \infty$, $\operatorname{Im} w<0$. We can eliminate those terms of order $w^{5 / 4}$ and $w^{3 / 4}$ by choosing $\beta^{\prime}=\gamma^{\prime}=0$. In order to eliminate those of order $1 / 4$, we find that

$$
K_{+}^{\prime}\left(\kappa_{1}\right) h_{+}^{\prime}\left(\kappa_{1}\right)+R_{1}^{\prime} K_{+}^{\prime}\left(-\kappa_{1}\right) h^{\prime}\left(-\kappa_{1}\right)-\frac{R_{3} K_{+}^{\prime}\left(-\kappa_{3}^{\prime}\right)}{h_{+}^{\prime}\left(-\kappa_{3}^{\prime}\right)}+\alpha^{\prime} \sqrt{2}=0 .
$$

The requirement that there be no waves in the smaller ducts from the right leads to the following two conditions:

and

$$
\begin{aligned}
& \frac{-4 a \mathrm{i} \sqrt{2} \alpha^{\prime}}{\pi}+\frac{R_{1}^{\prime} K_{+}^{\prime}\left(-\kappa_{1}\right) h_{+}^{\prime}\left(-\kappa_{1}\right)}{\kappa_{1}-\kappa_{2}} \\
& +\frac{3 R_{3} K_{+}^{\prime}\left(-\kappa_{3}^{\prime}\right)}{h_{+}^{\prime}\left(-\kappa_{3}^{\prime}\right)\left(\kappa_{3}^{\prime}-\kappa_{2}\right)}=\frac{K_{+}^{\prime}\left(\kappa_{1}\right) h_{+}^{\prime}\left(\kappa_{1}\right)}{\kappa_{2}+\kappa_{1}}
\end{aligned}
$$

$$
\frac{R_{1}^{\prime} K_{+}^{\prime}\left(-\kappa_{1}\right) h_{+}^{\prime}\left(-\kappa_{1}\right)}{\kappa_{1}-\kappa_{2}}-\frac{R_{3} K_{+}^{\prime}\left(-\kappa_{3}^{\prime}\right)}{h_{+}^{\prime}\left(-\kappa_{3}^{\prime}\right)\left(\kappa_{3}^{\prime}-\kappa_{2}\right)}=\frac{K_{+}^{\prime}\left(\kappa_{1}\right) h_{+}^{\prime}\left(\kappa_{1}\right)}{\kappa_{2}+\kappa_{1}} .
$$

Upon solving for $R_{1}^{\prime}, R_{3}$ and $\alpha^{\prime}$ we obtain

$$
\begin{aligned}
R_{1}^{\prime} & =\frac{K_{+}^{\prime}\left(\kappa_{1}\right) h_{+}^{\prime}\left(\kappa_{1}\right)\left(\kappa_{1}-\kappa_{2}\right)}{K_{+}^{\prime}\left(-\kappa_{1}\right) h_{+}^{\prime}\left(-\kappa_{1}\right)\left(\kappa_{1}+\kappa_{2}\right)} \frac{1-a \mathrm{i}\left(\kappa_{3}^{\prime}+\kappa_{1}\right) / \pi}{1-a \mathrm{i}\left(\kappa_{3}^{\prime}-\kappa_{1}\right) / \pi}, \\
R_{3} & =-\frac{2 \mathrm{i} a \kappa_{1}\left(\kappa_{3}^{\prime}-\kappa_{2}\right) h_{+}^{\prime}\left(-\kappa_{3}^{\prime}\right) h_{+}^{\prime}\left(\kappa_{1}\right) K_{+}^{\prime}\left(\kappa_{1}\right)}{\pi\left(\kappa_{1}+\kappa_{2}\right) K_{+}^{\prime}\left(-\kappa_{3}^{\prime}\right)\left[1-a \mathrm{i}\left(\kappa_{3}^{\prime}-\kappa_{1}\right) / \pi\right]}, \\
\alpha^{\prime} & =\frac{\sqrt{2} \kappa_{1} K_{+}^{\prime}\left(\kappa_{1}\right) h_{+}^{\prime}\left(\kappa_{1}\right)}{\left(\kappa_{1}+\kappa_{2}\right)\left[1-a \mathrm{i}\left(\kappa_{3}^{\prime}-\kappa_{1}\right) / \pi\right]} .
\end{aligned}
$$

It is a simple task to calculate $\left|R_{1}^{\prime}\right|$ and $\left|R_{3}\right|$. For since $K_{+}^{\prime}\left(\kappa_{1}\right) h_{+}^{\prime}\left(\kappa_{1}\right)$ is the conjugate of $K_{+}^{\prime}\left(-\kappa_{1}\right) h_{+}^{\prime}\left(-\kappa_{1}\right)$ and $\kappa_{1}^{2}-\left(\kappa_{3}^{\prime}\right)^{2}=\pi^{2} / 2 a^{2}$ we have

$$
\left|R_{1}^{\prime}\right|=\frac{\kappa_{1}-\kappa_{2}}{\kappa_{1}+\kappa_{2}} \sqrt{\frac{\left(\kappa_{1}+\kappa_{3}^{\prime}\right)\left(3 \kappa_{1}-\kappa_{3}^{\prime}\right)}{\left(\kappa_{1}-\kappa_{3}^{\prime}\right)\left(3 \kappa_{1}+\kappa_{3}^{\prime}\right)}} .
$$


Similarly, since $\left|h_{+}^{\prime}\left(\kappa_{1}\right) K_{+}^{\prime}\left(\kappa_{1}\right)\right|=(3 / 4)(\pi / 2)^{1 / 2}$ and $\left|h_{+}^{\prime}\left(-\kappa_{3}^{\prime}\right) / K_{+}^{\prime}\left(-\kappa_{3}^{\prime}\right)\right|$ $=4 \sqrt{2} / \sqrt{15 \pi}$, we find that

$$
\left|R_{3}\right|=\frac{2 \kappa_{1}}{\sqrt{\left(\kappa_{1}-\kappa_{3}^{\prime}\right)\left(3 \kappa_{1}+\kappa_{3}^{\prime}\right)}} \sqrt{\frac{\left(\kappa_{1}-\kappa_{2}\right)\left(\kappa_{2}-\kappa_{3}^{\prime}\right)}{\left(\kappa_{1}+\kappa_{2}\right)\left(\kappa_{2}+\kappa_{3}^{\prime}\right)}} .
$$

It should be remarked that although $\left|R_{3}\right|$ is the amplitude of the reflected wave of the second mode in the larger duct, it is not a reflection coefficient. To find the reflection coefficient of this mode, we recall the conservation relation

$$
\kappa_{1}\left[1-\left|R_{1}^{\prime}\right|^{2}\right]-\kappa_{3}^{\prime}\left|R_{3}\right|^{2}=\kappa_{2}\left[\left|T_{1}^{\prime}\right|^{2}+\left|T_{2}^{\prime}\right|^{2}\right] / 2 \text {. }
$$

It follows that the reflection coefficient for the first mode is $\left|R_{1}^{\prime}\right|$, whereas that coefficient for the second mode is $\left|R_{3}\right| \sqrt{\kappa_{3}^{\prime} / \kappa_{1}}$.

In order to determine the transmission coefficients, we are required to examine the representations of $\phi(x, y)$ for $0 \leqslant y \leqslant a$ and $a \leqslant y \leqslant 2 a$ separately. For $0 \leqslant y \leqslant a$ we have

$$
\begin{aligned}
\phi(x, y)= & \frac{\cos \pi y}{4 a}\left[\exp \left(\mathrm{i} \kappa_{1} x\right)+R_{1}^{\prime} \exp \left(-\mathrm{i} \kappa_{1} x\right)+R_{3} \cos \frac{3 \pi y}{4 a} \exp \left(-\mathrm{i} \kappa_{3} x\right)\right] \\
& +\frac{1}{2 \pi} \int_{\mathscr{L}} \frac{\cos \sigma(w) y}{\sigma(w) \cos 2 \sigma a}\left[\hat{\phi}_{y}(w) \sin a \sigma(w)\right. \\
& -\sigma(w) \hat{\phi}(w) \cos a \sigma(w)] \exp (\mathrm{i} w x) \mathrm{d} x
\end{aligned}
$$

where $\mathscr{L}$ is a path drawn parallel to the real axis and inside the strip $-\kappa_{5}<\operatorname{Im} w<0$. Since we now know $\alpha^{\prime}, \beta^{\prime}$ and $\gamma^{\prime}$, and therefore $\varepsilon_{1}^{\prime}(w)$ and $\varepsilon_{2}^{\prime}(w),(4.2)$ supplies us with $\hat{\phi}(w)$ and $\hat{\phi}_{y}(w)$, and (4.4) may be written as

$$
\begin{aligned}
\phi(x, y)= & \cos \frac{\pi y}{4 a}\left[\exp \left(\mathrm{i} \kappa_{1} x\right)+R_{1}^{\prime} \exp \left(-\mathrm{i} \kappa_{1} x\right)\right]+R_{3} \cos \frac{3 \pi y}{4 a} \exp \left(-\mathrm{i} \kappa_{3} x\right) \\
& +\frac{1}{4 \pi \sqrt{2}} \int_{\mathscr{L}}\left\{\frac{K_{+}^{\prime}\left(\kappa_{1}\right) h_{+}^{\prime}\left(\kappa_{1}\right)}{\mathrm{i}\left(w-\kappa_{1}\right)}\left[h_{+}^{\prime}(w)\left(\frac{\pi}{4 a \sigma}-1\right)-\frac{1}{h_{+}^{\prime}(w)}\left(\frac{\pi}{4 a \sigma}+1\right)\right]\right. \\
& +\frac{R_{1}^{\prime} K_{+}^{\prime}\left(-\kappa_{1}\right) h_{+}^{\prime}\left(-\kappa_{1}\right)}{\mathrm{i}\left(w+\kappa_{1}\right)}\left[h_{+}^{\prime}(w)\left(\frac{\pi}{4 a \sigma}-1\right)-\frac{1}{h_{+}^{\prime}(w)}\left(\frac{\pi}{4 a \sigma}+1\right)\right] \\
& +\frac{R_{3}^{\prime} K_{+}^{\prime}\left(-\kappa_{3}^{\prime}\right)}{\mathrm{i} h_{+}^{\prime}\left(-\kappa_{3}^{\prime}\right)\left(w+\kappa_{3}^{\prime}\right)}\left[h_{+}^{\prime}(w)\left(\frac{3 \pi}{4 a \sigma}+1\right)-\frac{1}{h_{+}^{\prime}(w)}\left(\frac{3 \pi}{4 a \sigma}+1\right)\right] \\
& \left.+\frac{\alpha^{\prime} \sqrt{2}}{\sigma}\left[h_{+}^{\prime}(w)-\frac{1}{h_{+}^{\prime}(w)}\right]\right\} \frac{\cos \sigma y \exp (\mathrm{i} w x)}{K_{+}(w) \cos \sigma a} \mathrm{~d} w .
\end{aligned}
$$

The integrand is single valued since $h_{+}^{\prime}(w)+1 / h_{+}^{\prime}(w)$ and $\left[h_{+}^{\prime}(w)-1 / h_{+}^{\prime}(w)\right] / \sigma(w)$ are single valued, and the remaining functions are obviously so.

We may now close the path $\mathscr{L}$ by a semicircle in the upper half-plane which passes between the zeros of $\cos \sigma \alpha$ to obtain the dominant term, that is the wave transmitted in the lower duct. When $x \rightarrow \infty, 0<y<a$ this gives us

$$
\frac{\pi}{4 \sqrt{2} a^{2} \kappa_{2}}\left\{\frac{K_{+}^{\prime}\left(\kappa_{1}\right) h_{+}^{\prime}\left(\kappa_{1}\right)}{\left(\kappa_{2}-\kappa_{1}\right)}\left[-\frac{h_{+}^{\prime}\left(\kappa_{2}\right)}{2}-\frac{3}{2 h_{+}^{\prime}\left(\kappa_{2}\right)}\right]\right.
$$




$$
\begin{aligned}
& +\frac{R_{1}^{\prime} K_{+}^{\prime}\left(-\kappa_{1}\right) h_{+}^{\prime}\left(-\kappa_{1}\right)}{\left(\kappa_{2}+\kappa_{1}\right)}\left[-\frac{h_{+}^{\prime}\left(\kappa_{2}\right)}{2}-\frac{3}{2 h_{+}^{\prime}\left(\kappa_{2}\right)}\right] \\
& +\frac{R_{3}^{\prime} K_{+}^{\prime}\left(-\kappa_{3}^{\prime}\right)}{\left(\kappa_{2}+\kappa_{3}^{\prime}\right) h_{+}^{\prime}\left(-\kappa_{3}^{\prime}\right)}\left[\frac{5}{2} h_{+}^{\prime}\left(\kappa_{2}\right)-\frac{1}{2 h_{+}^{\prime}\left(\kappa_{2}\right)}\right] \\
& \left.+\frac{2 \sqrt{2} a \alpha^{\prime} \mathrm{i}}{\pi}\left[h_{+}^{\prime}\left(\kappa_{2}\right)-\frac{1}{2 h_{+}^{\prime}\left(\kappa_{2}\right)}\right]\right\} \frac{\frac{\cos \pi y}{2 a} \exp \left(\mathrm{i} \kappa_{2} x\right)}{K_{+}\left(\kappa_{2}\right)} \\
& =T_{1}^{\prime} \cos \frac{\pi y}{2 a} \exp \left(i \kappa_{2} x\right) \text {. }
\end{aligned}
$$

$T_{1}^{\prime}$ may be simplified considerably by using the expressions we have found for $R_{1}^{\prime}, R_{3}$ and $\alpha^{\prime}$, and we obtain

$$
T_{1}^{\prime}=\frac{\pi \kappa_{1} K_{+}^{\prime}\left(\kappa_{1}\right) h_{+}^{\prime}\left(\kappa_{1}\right)\left\{\left[4\left(\kappa_{2}+\kappa_{3}^{\prime}\right)+\frac{5 i \pi}{a}\right] h_{+}^{\prime}\left(\kappa_{2}\right)+\left[12\left(\kappa_{2}+\kappa_{3}^{\prime}\right)+\frac{3 \mathrm{i} \pi}{a}\right] / h_{+}^{\prime}\left(\kappa_{2}\right)\right\}}{8 \sqrt{2} a^{2}\left(\kappa_{1}^{2}-\kappa_{2}^{2}\right)\left(\kappa_{1}+\kappa_{2}\right)\left(\kappa_{2}+\kappa_{3}^{\prime}\right)\left[1-a \mathrm{i}\left(\kappa_{3}^{\prime}-\kappa_{1}\right) / \pi\right] K_{+}\left(\kappa_{2}\right)} .
$$

For the case in which $x>0, a<y<2 a, \cos \sigma y$ in (4.4) is replaced by $\sin \sigma(2 a-y)$ and the terms in the square brackets by $[\cos \sigma a \hat{\phi} y-\sigma \sin \sigma a \hat{\phi}]$. Upon repeating the steps in the previous two paragraphs we obtain

$$
T_{2}^{\prime}=\frac{\pi \kappa_{1} K_{+}^{\prime}\left(\kappa_{1}\right) h_{+}^{\prime}\left(\kappa_{1}\right)\left\{\left[4\left(\kappa_{2}+\kappa_{3}^{\prime}\right)+\frac{5 \mathrm{i} \pi}{a}\right] h_{+}^{\prime}\left(\kappa_{2}\right)-\left[12\left(\kappa_{2}+\kappa_{3}^{\prime}\right)+\frac{3 \mathrm{i} \pi}{a}\right] / h_{+}^{\prime}\left(\kappa_{2}\right)\right\}}{8 \sqrt{2} a^{2}\left(\kappa_{1}^{2}-\kappa_{2}^{2}\right)\left(\kappa_{1}+\kappa_{2}\right)\left(\kappa_{2}+\kappa_{3}^{\prime}\right)\left[1-a \mathrm{i}\left(\kappa_{3}^{\prime}-\kappa_{1}\right) / \pi\right] K_{+}\left(\kappa_{2}\right)} .
$$

That is, $T_{1}^{\prime}$ and $T_{2}^{\prime}$ differ by the algebraic sign before the term containing $1 / h_{+}^{\prime}\left(\kappa_{2}\right)$. From $T_{1}^{\prime}$ and $T_{2}^{\prime}$ it is a simple task to determine their magnitudes and we obtain

and

$$
\begin{aligned}
\left|T_{1}^{\prime}\right|^{2}= & \frac{\kappa_{1}^{2}\left(\kappa_{1}+\kappa_{3}^{\prime}\right)}{2\left(\kappa_{1}+\kappa_{2}\right)\left(\kappa_{2}+\kappa_{3}^{\prime}\right)\left(3 \kappa_{1}+\kappa_{3}^{\prime}\right)} \\
& \times\left[4\left(3 \kappa_{2}+\kappa_{3}^{\prime}\right)+\sqrt{15}\left(2 \kappa_{2} \cos 2 v^{\prime}-\frac{\pi}{a} \sin 2 v^{\prime}\right)\right]
\end{aligned}
$$

$$
\begin{aligned}
\left|T_{2}^{\prime}\right|^{2}= & \frac{\kappa_{1}^{2}\left(\kappa_{1}+\kappa_{3}^{\prime}\right)}{2\left(\kappa_{1}+\kappa_{2}\right)\left(\kappa_{2}+\kappa_{3}^{\prime}\right)\left(3 \kappa_{1}+\kappa_{3}^{\prime}\right)} \\
& \times\left[4\left(3 \kappa_{2}+\kappa_{3}^{\prime}\right)-\sqrt{15}\left(2 \kappa_{2} \cos 2 v^{\prime}-\frac{\pi}{a} \sin 2 v^{\prime}\right)\right],
\end{aligned}
$$

where $v^{\prime}=\arg h_{+}^{\prime}\left(\kappa_{2}\right)$.

We can now verify the conservation condition (4.3) and observe that

$$
\sqrt{\frac{\kappa_{2}}{2 \kappa_{1}}}\left|T_{1}^{\prime}\right| \text { and } \sqrt{\frac{\kappa_{2}}{2 \kappa_{1}}}\left|T_{2}^{\prime}\right|
$$

are the transmission coefficients in the small ducts. We further note that for $a k=3 \pi / 4$, that is the cut-off for the second mode in the left duct, $\kappa_{2}=\pi \sqrt{5} / 4 a$, and hence

$$
v\left(\frac{\sqrt{5} \pi}{4 a}\right)=v^{\prime}\left(\frac{\sqrt{5} \pi}{4 a}\right)+\frac{1}{2} \arcsin \frac{2}{3}=v^{\prime}\left(\frac{\sqrt{5} \pi}{4 a}\right)+\frac{1}{2} \arccos \frac{\sqrt{5}}{3} \text {. }
$$


Hence for $a k=3 \pi / 4,\left|T_{1}\right|=\left|T_{1}^{\prime}\right|$ and $\left|T_{2}\right|=\left|T_{2}^{\prime}\right|$ upon comparing and simplifying $T_{1}, T_{1}^{\prime}, T_{2}, T_{2}^{\prime}$. Further without the need for $v$ and $v^{\prime}$, we can show directly that $\left|R_{1}\right|=\left|R_{1}^{\prime}\right|$ at $a k=3 \pi / 4$. However, the slopes of $\left|R_{1}\right|=\left|R_{1}^{\prime}\right|$ at $a k=3 \pi / 4$ are not continuous and in fact the slope of $\left|R_{1}^{\prime}\right|$ is vertical when $a k \rightarrow 3 \pi / 4+0$, whereas the slope of $R_{1}$ is not. The same is true also for $\left|T_{1}\right|$ and $\left|T_{1}^{\prime}\right|$ as well as $\left|T_{2}\right|=\left|T_{2}^{\prime}\right|$. Figure 2 is a graph of $\left|R_{1}\right|$ and Fig. 3 is a graph of $\left|R_{1}^{\prime}\right|$. These graphs do not agree with those in [9].

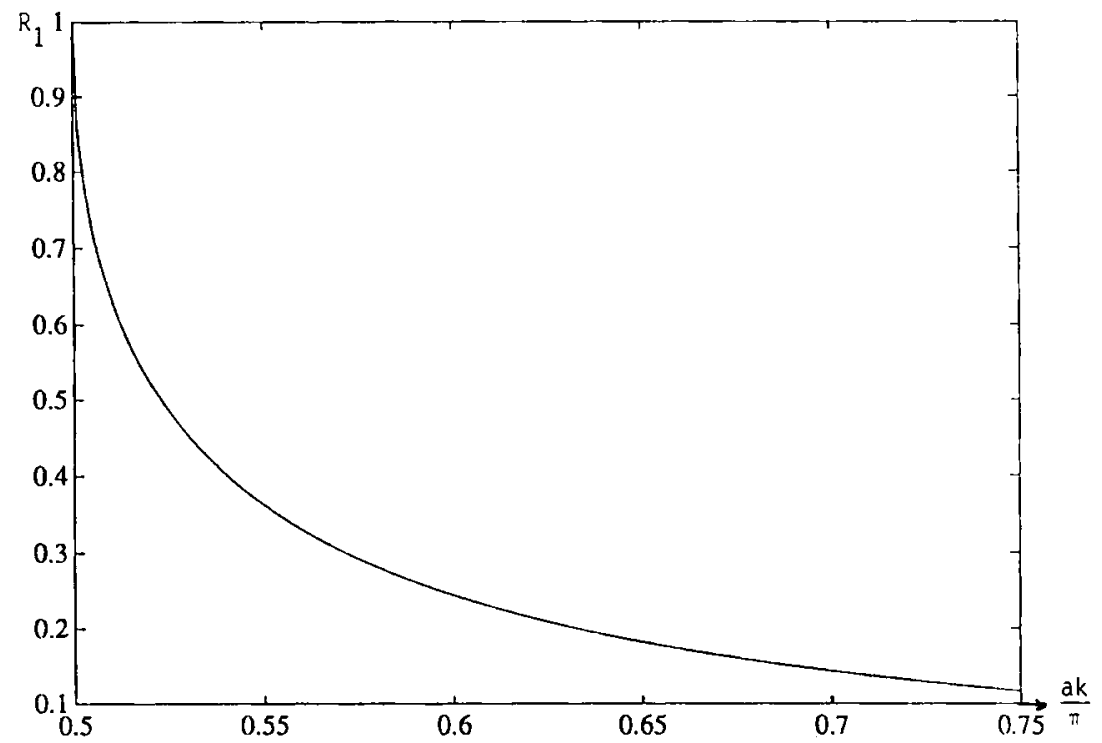

Fig. 2.

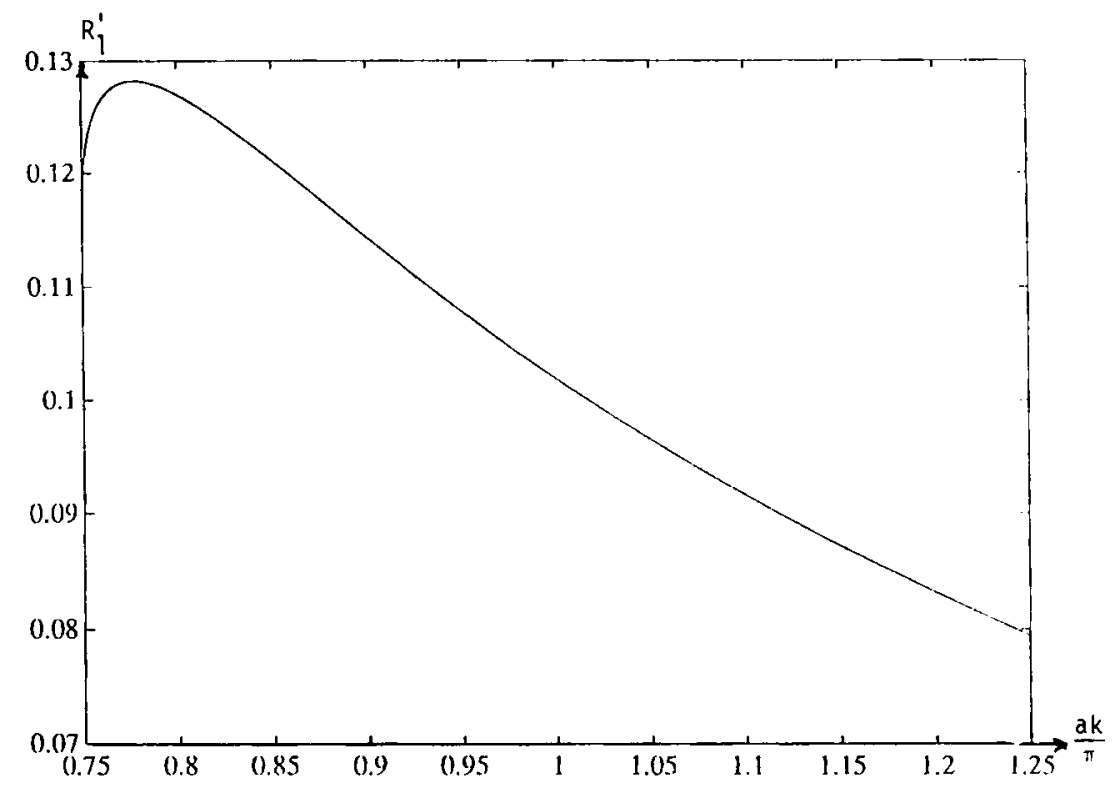

Fig. 3 


\section{The case $k$ complex}

We have solved the two problems which we described in Section 2 when $k$ is real and positive. At this point we wish to note the modifications which are necessary when $k$ is complex with $\operatorname{Im} k>0$. We find that the solutions are of the same respective forms if we employ $G^{(1)}\left(x, y ; x^{\prime}, y^{\prime}\right)$ or $G^{(2)}\left(x, y ; x^{\prime}, y^{\prime}\right)$ for the two cases at hand. However, some Wiener-Hopf aspects are modified for the case which employs $G^{(1)}\left(x, y ; x^{\prime}, y^{\prime}\right)$. We shall see that we are required to work in the strip $-\operatorname{Re} \sqrt{\left(9 \pi^{2} / 16 a^{2}\right)-k^{2}}$ $<\operatorname{Im} w<-\operatorname{Im} \sqrt{k^{2}-\left(\pi^{2} / 4 a^{2}\right)}$, where both $\operatorname{Re} \sqrt{\left(9 \pi^{2} / 16 a^{2}\right)-k^{2}}$ and $\operatorname{Im} \sqrt{k^{2}-\left(\pi^{2} / 4 a^{2}\right)}$ are positive. For the case which employs $G^{(2)}\left(x, y ; x^{\prime}, y^{\prime}\right)$ we are required to work in the strip $-\operatorname{Re} \sqrt{\left(25 \pi^{2} / 16 a^{2}\right)}-k^{2}<\operatorname{Im} w<$ $-\operatorname{Im} \sqrt{k^{2}-\left(9 \pi^{2} / 16 a^{2}\right)}$, where now $\operatorname{Re} \sqrt{\left(25 \pi^{2} / 16 a^{2}\right)-k^{2}}$ and $\operatorname{Im} \sqrt{\left(9 \pi^{2} / 16 a^{2}\right)-k^{2}}$ are positive.

In order to see how these inequalities are derived, we note that the unilateral Fourier transforms of $\phi(x, a-0)$ and $\left.\phi_{y}(x, y)\right|_{y=a+0}, x>0$ are now analytic in the lower half-plane $\operatorname{Im} w<-\operatorname{Im} \sqrt{k^{2}-\left(\pi^{2} / 4 a^{2}\right)}$. In the case that we employ the Green's function $G^{(1)}\left(x, y ; x^{\prime}, y^{\prime}\right)$ we find that its bilateral Fourier transform is analytic in the strip $-\operatorname{Re} \sqrt{\left(9 \pi^{2} / 16 a^{2}\right)-k^{2}}<\operatorname{Im} w<-\operatorname{Im} \sqrt{k^{2}-\left(\pi^{2} / 16 a^{2}\right)}$, whereas the unilateral Fourier transforms of $\psi(x, a)$ and $\left.\psi_{y}(x, y)\right|_{y=a}$ are analytic in the upper half-plane $-\operatorname{Re} \sqrt{\left(9 \pi^{2} / 16 a^{2}\right)-k^{2}}$. This provides us with analyticity in the strip $-\operatorname{Re} \sqrt{\left(9 \pi^{2} / 16 a^{2}\right)-k^{2}}<\operatorname{Im} w<-\operatorname{Im} \sqrt{k^{2}-\left(\pi^{2} / 4 a^{2}\right)}$. The case for which we employ $G^{(2)}\left(x, y ; x^{\prime}, y^{\prime}\right)$ gives us a similar inequality.

In the formulation used by Lüneburg and Hurd [9], there is a strip $-\operatorname{Im} \sqrt{k^{2}-\left(\pi^{2} / 16 a^{2}\right)}<\operatorname{Im} w<\operatorname{Im} \sqrt{k^{2}-\left(\pi^{2} / 16 a^{2}\right)}$ (which they do not mention). That is, their Green's function $G\left(x, y ; x^{\prime}, y^{\prime}\right)$ now has a bilateral Fourier transform analytic in the above strip, whereas the unilateral Fourier transforms of $\phi(x, a-0)$ and $\left.\phi_{y}(x, y)\right|_{y=a+0}, \quad x>0$ are analytic in the lower half-plane $\operatorname{Im} w<\operatorname{Im} \sqrt{k^{2}-\left(\pi^{2} / 16 a^{2}\right)}$. The truncated $\phi(x, a)$ and $\phi_{y}(x, a), x<0$ are now analytic in the upper half-plane $\operatorname{Im} w>-\operatorname{Im} \sqrt{k^{2}-\left(\pi^{2} / 16 a^{2}\right)}$ and hence there is a common strip of analyticity in their development, namely $-\operatorname{Im} \sqrt{k^{2}-\left(\pi^{2} / 16 a^{2}\right)}$ $<\operatorname{Im} w<\operatorname{Im} \sqrt{k^{2}-\left(\pi^{2} / 16 a^{2}\right)}$ where $\operatorname{Im} \sqrt{k^{2}-\left(\pi^{2} / 16 a^{2}\right)}>0$. The meaning of their expressions 'upper and lower half-planes' is not clear. The limits in their integral in equation (1.10) of their paper [9] should be replaced by a path $P$ which is a line parallel to the real axis of the $w$ plane, drawn in the strip which we have described here. What we have called $h(w)$ and they call $t(w)$ differ by some factors which appear in some other parts of our development. Finally, the incident field has been omitted in (1.32c) and (1.32d) since they had made the tacit assumption that their $\alpha_{1}$ (our $\left.\sqrt{k^{2}-\left(\pi^{2} / 16 a^{2}\right)}\right)$ is real. Upon taking advantage of the strip of analyticity which is now available in their development, their limits $(-\infty, \infty)$ should now be replaced by $P$ in $(1.32 \mathrm{a}, \mathrm{b}, \mathrm{c}, \mathrm{d})$.

The magnitudes of the reflection coefficients calculated in [9] are based on the tacit assumption that $k$ is real, although their formulation of the problem relies on the fact that $k$ has a positive imaginary part. These magnitudes, however, agree with our results when $k$ is real. Our transmission coefficients are expressed in considerably simpler form and only require the numerical calculation of the phase angles $v$ and $v^{\prime}$. 


\section{References}

1. Heins, A. E., 'Systems of Wiener-Hopf integral equations and their applications to some boundary value problems in electromagnetic theory', Proc. Symp. Appl. Math., Am. Math. Soc., Vol. 2, 1950, pp. 76-81.

2. Heins, A. E., 'The Green's function for periodic structures on diffraction theory with an application to parallel plate media', I and II, J. Math. Mech., 6, 401-426; 629-639 (1957).

3. Heins, A. E., 'The Sommerfeld half-plane problem revisited, II': The factoring of a matrix of analytic functions, Math. Meth. in the Appl. Sci., 5, 14-21 (1983).

4. Heins, A. E., 'The Sommerfeld half-plane problem revisited, III: parallel plate media with mixed boundary conditions', Math. Meth. in the Appl. Sci., 5, 257-275 (1983).

5. Heins, A. E. and Pond, J.M., 'The Sommerfeld half-plane problem revisited, IV: variations on a theme of Carlson and Heins', Math. Meth. in the Appl. Sci., 10, 303-328 (1988).

6. Hille, E., Analytic Function Theory, Vol. 1, Ginn and Co., Boston, New York, Chicago, Atlanta, Dallas, Palo Alto, Toronto, London, 1959.

7. Lüneburg, E. and Hurd, R. A., 'Diffraction by an infinite set of hard and soft parallel half-planes', Canadian J. Phys., 60, 1-9 (1982).

8. Lüneburg, E. and Hurd, R. A., 'Diffraction by an infinite set of soft/hard parallel half-planes', Radio Science, 17, 453-462 (1982).

9. Lüneburg, E. and Hurd, R. A., 'Two waveguide bifurcation problems', $Z A M M, 65,551-559$ (1985).

10. Meister, E., 'Zum Dirichlet-Problem der Helmholtzschen Schwingungsgleichung für ein Gestaffeltes Streckengitter', Arch. f. Rat. Mech. and Anal., 10, 67-100 (1962).

11. Schwinger, J. S. and Saxon, D. S., Discontinuities in Wave Guides (Notes on Lectures by Julian Schwinger), Gordon and Breach, New York, London, Paris, 1968. 